\title{
Ascitis quilosa tras colecistectomía. Presentación de un caso
}

\author{
Alberto Vilar-Tabanera ${ }^{1}$, Francisco García-Angarita ${ }^{1}$, \\ Elena Mendía-Conde ${ }^{1}$ y Joaquín Gómez-Ramírez ${ }^{1}$
}

\section{Chylous ascites after cholecystectomy. Case report and literatura review}

Introduction: Chylous ascites is defined as the presence of lymph fluid in the peritoneal cavity. It is a rare complication after abdominal surgery; only 5 previously reported cases were found after cholecystectomy. Aim: Present a case report and a literature review. Materials and Method: Case report of a 77 year old male who underwent an elective cholecystectomy due to acute cholecystitis. Chyloperitoneum showed up 21 days after surgery. Results: We performed a percutaneous drainage and $5 \mathrm{~L}$ of fluid were removed in 24 hours. We started treatment with subcutaneous Octreotide and total parenteral nutrition. After 3 days drain output decreased and we started a medium-chain triglycerides diet with good progress. The outcome of $60 \%$ of the 5 previous case reports of chyloperitoneum after cholecystitis, were successful with conservative management, surgical intervention was needed in one patient and a transjugular intrahepatic portosystemic shunt (TIPSS) was placed in another patient. Conclusion: Chylous ascites is a rare complication after surgery, there are only 5 previously case reports after cholecystectomy. Conservative management has to be the first option and in case of persistence another therapy has to be considered.

Key words: chylous ascites; chyloperitoneum; cholecystectomy.

\section{Resumen}

Introducción: La ascitis quilosa es la presencia de líquido linfático en la cavidad peritoneal. Como consecuencia de una cirugía abdominal es muy infrecuente, encontrando 5 casos previos en la literatura revisada tras colecistectomía. Objetivo: Presentar un caso clínico de ascitis quilosa poscolecistectomía, su manejo y una revisión de la literatura. Materiales y Métodos: Varón de 77 años, quiloperitoneo 21 días después de realización de colecistectomía programada por colecistitis aguda. Resultados: Se realiza drenaje percutáneo con débito de $5 \mathrm{~L}$ en 24 horas, se inicia octreótido subcutáneo y nutrición parenteral total. Al tercer día disminuye el débito por el drenaje, por lo que se inicia dieta rica en triglicéridos de cadena media con buena evolución posterior. De los 5 casos previos tras colecistectomía, el $60 \%$ se resolvió con tratamiento conservador, un paciente precisó reintervención y otro colocación de un shunt portosistémico intrahepático trasnyugular (TIPSS). Conclusión: La ascitis quilosa es una complicación postquirúrgica infrecuente, encontrando solo 5 casos previos tras colecistectomía. Inicialmente el manejo debe ser conservador, en caso de persistencia se deben valorar otras medidas.

Palabras clave: ascitis quilosa; quiloperitoneo; colecistectomía.
'Departamento de Cirugía General y del Aparato Digestivo. Hospital Universitario Ramón y Cajal. Madrid, España

Recibido el 5 de junio de 2018 y aceptado para publicación el 24 de julio de 2018.

\section{Correspondencia a:} Alberto Vilar-Tabanera alb.vilartabanera@gmail.com

\section{Introducción}

La ascitis quilosa o quiloperitoneo se define como la presencia o acumulación de líquido linfático en la cavidad peritoneal. Este líquido suele presentar un aspecto lechoso y ser rico en triglicéridos, proteínas y linfocitos ${ }^{1}$. Como consecuencia de una cirugía abdominal previa es muy poco frecuente y su incidencia descrita en la literatura es escasa. El mecanismo fisiopatológico por el que se produce no está del todo aclarado, así como su diagnóstico y tratamiento posterior. Se presenta el caso de un paciente que presentó dicha patología tras una colecistectomía, su manejo y por último una revisión de la literatura existente.

\section{Caso clínico}

Se trata de un varón de 77 años, como antecedentes presenta hipertensión arterial y sobrepeso. Se 
realizó colecistectomía laparoscópica programada por colecistolitiasis sintomática (episodio previo de colecistitis aguda) que es convertida a cirugía abierta por importante plastrón inflamatorio; durante la intervención se decide realizar una colecistectomía parcial. El paciente presenta una fuga biliar en el postoperatorio manejada con CPRE y colocación de prótesis biliar plástica, debido a buena evolución es dado de alta. Tres semanas después de la intervención acude a urgencias por distensión abdominal, se le realiza tomografía axial computarizada (TC) abdominal que muestra abundante ascitis (Figura 1), se realiza paracentesis diagnóstica en la que se aprecia salida abundante de líquido lechoso (Figura 2), en el análisis bioquímico destaca un aumento de triglicéridos y proteínas. Ante el diagnóstico de

Figura 1. TC abdominal que muestra abundante ascitis y colección en el lecho de la colecistectomía.

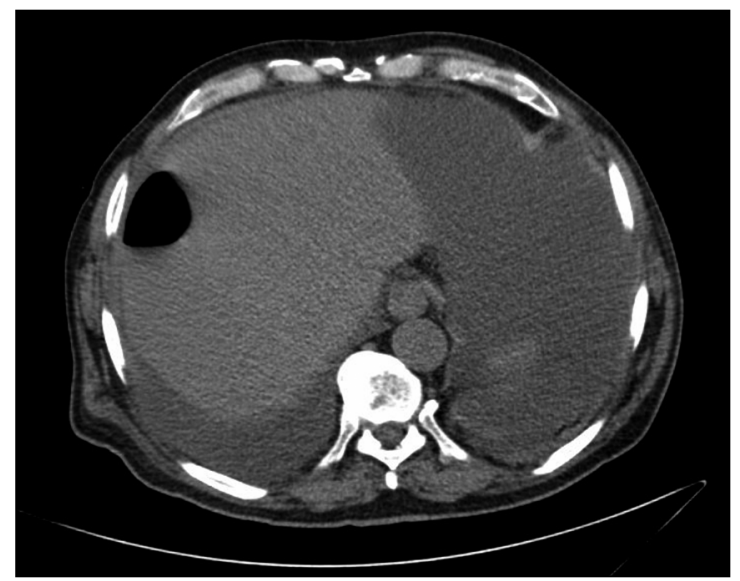

Figura 2. Líquido quiloso abdominal obtenido tras drenaje percutáneo.

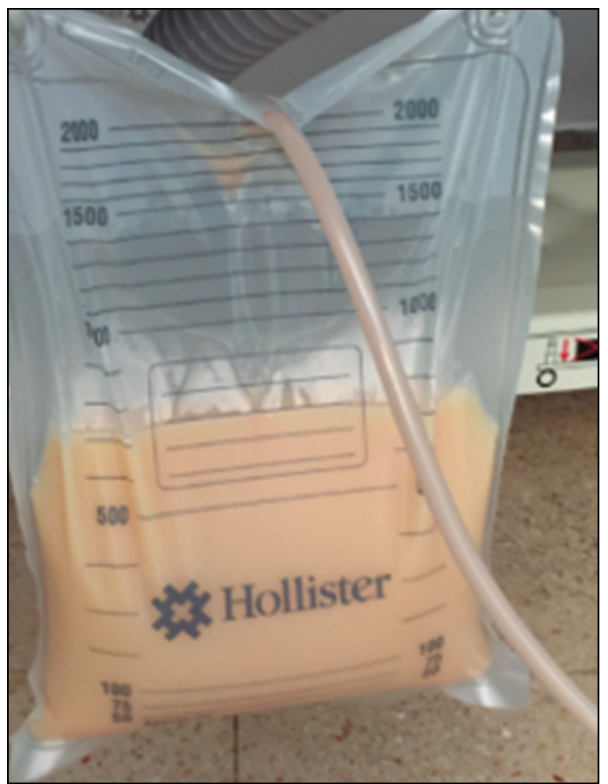

ascitis quilosa, se inicia nutrición parenteral total (NTP) y se coloca drenaje percutáneo con salida de $5 \mathrm{~L}$ de líquido las primeras 24 horas. Se inicia tratamiento con octreótido subcutáneo cada 8 horas. A los 3 días de inicio de NTP disminuye el débito y el líquido pasa a ser seroso, por lo que de acuerdo con el servicio de endocrinología se inicia dieta con triglicéridos de cadena media. El paciente evoluciona favorablemente siendo dado de alta a los 15 días.

\section{Discusión}

La ascitis quilosa es una complicación postquirúrgica muy infrecuente, como complicación tras colecistectomía es excepcional, encontrando 5 casos previos en la literatura (Tabla 1). La primera descripción de ascitis quilosa se realizó en 1691 por Richard Morton tras realizar una paracentesis a un niño con tuberculosis diseminada ${ }^{2}$.

La etiología de esta acumulación de líquido linfático suele ser la disrupción u obstrucción de los conductos linfáticos a nivel abdominal o torácico. Esta alteración de los conductos linfáticos puede ser consecuencia de tumores, fibrosis ocasionada por procesos inflamatorios, infecciones, causas congénitas, traumáticas tanto quirúrgicas como no quirúrgicas. En países desarrollados la causa más frecuente son las neoplasias y la cirrosis, sin embargo, la ascitis asociada a la cirrosis tiene unas características clínicas, un mecanismo y un manejo distinto a la ascitis quilosa. La ascitis causada por la cirrosis se debe, principalmente, a la hipertensión portal (HTP), la principal consecuencia es un aumento de la filtración de líquido desde los sinusoides hepáticos y capilares esplácnicos al intersticio. Inicialmente, es compensado por un aumento del retorno de líquido al torrente circulatorio a través de la circulación linfática, aumentando el flujo de linfa a través del conducto torácico de forma significativa respecto a los individuos sanos. Sin embargo, a medida que la enfermedad progresa, la formación de ascitis sobrepasa la capacidad de drenaje del conducto torácico, lo que determina la filtración de líquido a la cavidad peritoneal. Además, se produce una vasodilatación arterial esplácnica mediada por el óxido nítrico con un acúmulo mayor del volumen arterial total en el territorio esplácnico. Los barorreceptores arteriales lo interpretan como una disminución del volumen arterial y como respuesta activan el sistema renina-angiotensina-aldosterona para aumentar el volumen plasmático. Es importante destacar que los pacientes cirróticos también pueden presentar una ascitis quilosa asociada por lo que pueden coexistir ambos procesos, por ejemplo 
Tabla 1. Quiloperitoneo tras colecistectomía. Reporte de casos previos

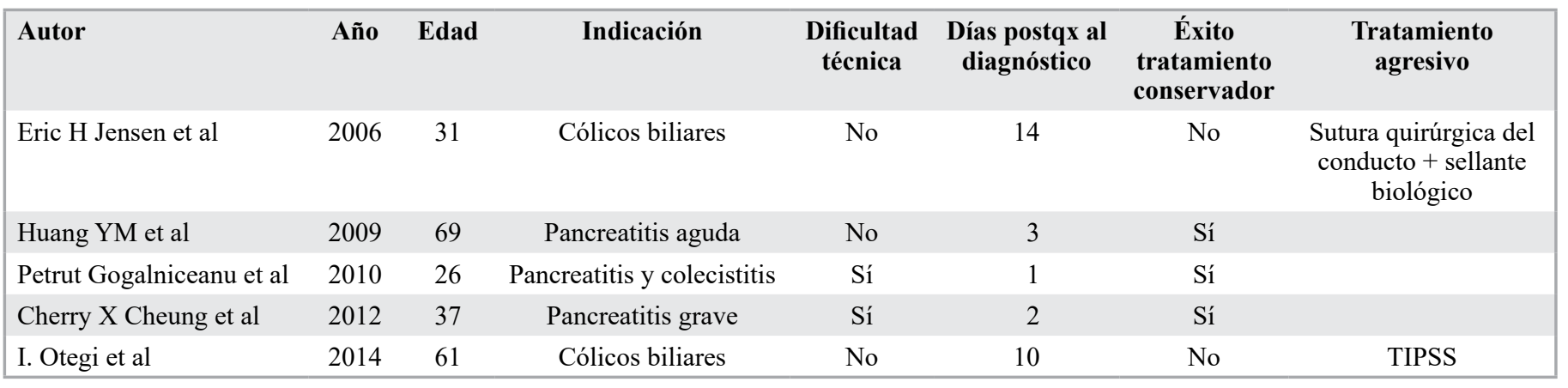

tras una intervención quirúrgica, teniendo en estos casos un pronóstico y evolución más desfavorable que en individuos previamente sanos. Para poder distinguir entre ambos procesos es imprescindible el análisis del líquido ascítico, ya que presentan unas características distintas (Tabla 2). El tratamiento de la ascitis por hipertensión portal se basa en la restricción de sal, diuréticos y en última instancia derivaciones portosistémicas.

En cambio, en países subdesarrollados suelen estar causadas principalmente por infecciones como tuberculosis y filariasis. Se trata de una patología muy infrecuente con una incidencia real desconocida $^{3}$.

A pesar de que la patología biliar es muy prevalente en nuestro medio y a que existe un aumento en el número de colecistectomías realizadas en las últimas décadas, los casos de ascitis quilosa después de una colecistectomía tanto abierta como laparoscópica son infrecuentes, encontrando únicamente 5 publicaciones previas de casos clínicos aislados, proponiéndose en una de ellas a la fibrosis de los conductos linfáticos ocasionada por el antecedente de pancreatitis aguda grave. Como complicación postquirúrgica es más habitual, aunque anecdótica, en otro tipo de intervenciones y de patologías, como en cirugías de la aorta abdominal o en las que se realiza linfadenectomía retroperitoneal.

Son importantes las consecuencias nutricionales e inmunológicas que este proceso supone para el paciente. El líquido linfático es rico en proteínas, en lípidos, principalmente triglicéridos y en linfocitos, por lo que van a ocasionar hipoproteinemia, depleción de reservas de grasa y de vitaminas liposolubles y linfopenia con el correspondiente aumento del riesgo de infecciones.

En cuanto al diagnóstico, la anamnesis adquiere especial relevancia para averiguar la etiología. En la exploración destacan los signos habituales de un
Tabla 2. Comparativa análisis del líquido ascítico

\begin{tabular}{|lll|}
\hline & Ascitis quilosa & Ascitis por HTP \\
Aspecto del líquido & Lechoso & Ambarino \\
PH & Alcalino & Alcalino \\
Proteínas & $>2,5 \mathrm{~g} / \mathrm{dl}$ & $<2,5 \mathrm{~g} / \mathrm{dl}$ \\
Leucocitos & $>250($ linfocitos $)$ & $<250$ \\
Triglicéridos & $>200 \mathrm{mg} / \mathrm{dl}$ & $<200 \mathrm{mg} / \mathrm{dl}$ \\
SAAG $($ Serum ascites albumin gradient $)$ & $<1,1 \mathrm{~g} / 100 \mathrm{ml}$ & $>1,1 \mathrm{~g} / 100 \mathrm{ml}$ \\
\hline
\end{tabular}

paciente con ascitis, distensión abdominal, matidez a la percusión, no suelen estar presentes signos de irritación peritoneal. La realización de pruebas de imagen (ecografía abdominal o TC) es útil para aclarar la etiología. Para el diagnóstico hay que realizar una paracentesis del líquido que suele presentar aspecto lechoso y realizar análisis bioquímico del mismo. En la bioquímica destaca una cifra de triglicéridos superior a $200 \mathrm{mg} / \mathrm{dl}$, aumento de proteínas y PH alcalino.

La linfografía con Tecnecio es la técnica más sensible y específica para identificar el punto de fuga de líquido linfático hacia el peritoneo, en cambio debido a la dificultad técnica y que no se encuentra exenta de riesgos, se reserva para casos refractarios a tratamiento inicial o persistente ${ }^{4}$.

El tratamiento recomendado en estos pacientes depende del estado general del paciente y de la etiología. Como norma general, inicialmente, se debe optar por el tratamiento conservador, mejorando el estado nutricional del paciente, drenando el líquido abdominal y disminuyendo el débito de la fístula ${ }^{5}$. Por lo tanto, será necesario introducir o bien una dieta sin triglicéridos de cadena larga utilizando triglicéridos de cadena media, que pasan directamente a la circulación sistémica o iniciar nutrición parenteral total (de elección en casos con abundante 
débito de líquido linfático). Se recomienda drenaje percutáneo para drenar el líquido intraabdominal y controlar el débito. Se han utilizado somatostatina y análogos de la misma como el octreótrido con el fin de reducir el débito, ya que disminuyen la absorción de grasas y el flujo esplácnico. La Somatostatina tiene una vida media muy corta (1-2 minutos) por lo que debe ser pautada en perfusión continua, en cambio el octreótrido tiene una vida media más larga por lo que puede pautarse de forma subcutánea cada 8 horas.

En casos de persistencia del quiloperitoneo, fracaso del manejo conservador o mala evolución del paciente se puede realizar una linfografía con tecnecio y su embolización con colocación de coils. Desde el punto de vista quirúrgico se puede plantear la reintervención y sutura directa del defecto o ligadura del conducto, pudiendo durante la intervención aplicar en el lecho pegamentos biológicos. Otra técnica utilizada en el manejo de estos pacientes ha sido la realización de shunt portosistémico intrahepático trasnyugular (TIPSS) principalmente en pacientes que presentan hipertensión portal. Una medida más agresiva que puede realizarse en pacientes con ascitis refractaria, es la colocación de shunt peritoneo-venosos ${ }^{6}$.

En todos los casos clínicos aislados publicados entre 2006 y 2014 inicialmente se optó por el tratamiento conservador siendo satisfactorio en 3 casos. En los otros dos casos en los que el tratamiento conservador no consiguió controlar la ascitis, se optó en un caso por la reintervención y cierre primario del conducto. En el otro caso refractario a tratamiento conservador se colocó un TIPSS siendo en ambos casos la evolución favorable. En los 3 casos manejados de forma conservadora en la literatura, el diagnóstico se llevó a cabo en las primeras 24-72 horas después de la intervención, en cambio en los dos casos en los que no fue efectivo el manejo conservador, la fuga se presentó en el décimo y en el decimocuarto día postoperatorio respectivamente, pudiendo estar en relación el diagnóstico y tratamiento precoz con la posibilidad de éxito del tratamiento conservador ${ }^{7-10}$.

Por tanto, al parecer el tratamiento conservador en este contexto consigue controlar la ascitis en un porcentaje importante de pacientes (llegando al 67\% si incluimos nuestro paciente), además es importante destacar que no se ha registrado mortalidad en ninguno de los 5 casos previos ni en nuestro paciente. Por tanto, según estos resultados y de acuerdo con la literatura previa, parece que la ascitis quilosa posquirúrgica presenta mejor pronóstico y evolución que en otras etiologías.

\section{Conclusión}

La ascitis quilosa como complicación postquirúrgica es infrecuente, siendo más frecuente tras cirugía aórtica y retroperitoneal. Se han encontrado cinco casos previos tras colecistectomía en la literatura. Inicialmente el manejo debe ser conservador y en caso de persistencia se pueden valorar medidas más agresivas.

\section{Responsabilidades éticas}

Protección de personas y animales. Los autores declaran que para esta investigación no se han realizado experimentos en seres humanos ni en animales.

Confidencialidad de los datos. Los autores declaran que en este artículo no aparecen datos de pacientes.

Conflictos de interés: no hay.

\section{Referencias}

1. Cárdenas A, Chopra S. Chylous ascites. American Journal of Gastroenterology 2002;97:1896-900.

2. Aalami OO, Allen DB, MD, Organ Jr CH. Chylous ascites: A collective review, Surgery 2000;128:761-78.

3. Browse NL, Wilson NM, Russo F, AlHassan H, Allen DR. Aetiology and treatment of chylous ascites. Br J Surg. 1992;79:1145-50.

4. Pui MH, Yueh TC. Lymphoscintigraphy in chyluria, chyloperitoneum and chylothorax. J Nucl Med. 1998;39:1292-6.

5. Leibovich I, Mor Y, Golomb J, Ramon

$\mathrm{J}$. The diagnosis and management of postoperative chylous ascites. J Urol. 2002;167:449-57.

6. Voros D, Hadziyannis S. Successful management of postoperative chylous ascites with a peritoneojugular shunt. J Hepatol. 1995;22:380.

7. Jensen EH, Weiss CA 3rd. Management of chylous ascites after laparoscopic cholecystectomy using minimally invasive techniques: a case report and literature review. Am Surg. 2006;72:60-3.
8. Huang YM, Chen JH, Liu SH, Lin MT. Chlye leakage after laparoscopic cholecystectomy for acute biliary pancreatitis: a case report. Hepatogastroenterology 2009;56:39-42.

9. Gogalniceanu P, Purkayastha S, Salding D, Zacharakis E. Chyle leak following laparoscopic cholecystectomy: a rare complication. Ann R Coll Surg Engl. 2010;92:12-4.

10. Cheung CX, Kelly ME, El Tayeb O, Torreggiani WC, Ridgway OF. Chylous ascites post open cholecystectomy after severe pancreatitis. JOP. 2012;13:278-81. 